\title{
AN EXAMPLE OF A CARATHÉODORY COMPLETE BUT NOT FINITELY COMPACT ANALYTIC SPACE
}

\author{
MAREK JARNICKI, PETER PFLUG, AND JEAN-PIERRE VIGUÉ
}

(Communicated by Clifford J. Earle, Jr.)

\begin{abstract}
An analytic space is given which is $c_{X}$-complete but not $c_{X}$-finitely
\end{abstract} compact.

\section{INTRODUCTION}

If $X$ is a (connected) complex analytic space, the Carathéodory pseudodistance $c_{X}$ on $X$ is given by

$$
c_{X}(x, y)=\sup \{\omega(f(x), f(y)): f \in \mathscr{O}(X, \Delta)\},
$$

where $\mathscr{O}(X, \Delta)$ denotes the set of all holomorphic mappings from $X$ to the open unit disc $\Delta \subset \mathbb{C}$ and where $\omega$ is the hyperbolic (Poincaré) distance on $\Delta$. Now let us consider an analytic space $X$ for which $c_{X}$ is a distance defining the topology of $X$ (cf. [2,7]). If the $c_{X}$-balls are relatively compact in $X$, i.e., $X$ is finitely compact with respect to $c_{X}$, then it is clear that $X$ is $c_{X}$-complete. The problem of the equivalence of these two notions has been raised by Kobayashi [4] (see also [1]). A positive answer for plane domains was given by Selby [5] and Sibony [6]. (We thank C. J. Earle for showing us the paper of Selby.) The purpose of this note is to construct an analytic space $X, c_{X}$-complete but not finitely compact with respect to $c_{X}$. The case of domains in $\mathbb{C}^{n}, n>1$, still remains open.

\section{Construction OF THE EXAMPLE}

For every integer $n>0$ let

$$
p_{k}^{n}:=\left(1-\frac{1}{n+1}\right) \exp \left(\frac{2 \pi i k}{n+1}\right) \in \Delta, \quad 0 \leq k \leq n .
$$

We construct a connected analytic space $X$ in the following way:

Let $\left(D_{j}\right)_{j=0}^{\infty}$ be a sequence of copies of $\Delta$, i.e., $D_{j}:=\Delta$, and let $q_{k}^{n} \in D_{0}$ be defined by $q_{k}^{n}:=p_{k}^{n}, n>0,0 \leq k \leq n$. Moreover, for every $n>0$, let $x_{k}^{n} \in D_{n}$ be given by $x_{k}^{n}:=p_{k}^{n}, 0 \leq k \leq n$.

Received by the editors October 10, 1991.

1991 Mathematics Subject Classification. Primary 32H15.

The third author is a membre de l'URA, CNRS D 1322 "Groupes de Lie et géométrie". 
Then the analytic space $X$ is obtained by patching together $D_{n}$ and $D_{0}$, $n>0$, by identifying the points $q_{k}^{n}$ and $x_{k}^{n}, 0 \leq k \leq n$.

Topologically, $X$ is the quotient of $\dot{\bigcup}_{n=0}^{\infty} D_{n}$ by the equivalence relation $\mathscr{R}: q_{k}^{n} \mathscr{R} x_{k}^{n}, n>0, k=0, \ldots, n$. The analytic structure of $X$ is defined by gluing $D_{0}$ and $D_{n}, n>0$, transversally at $q_{k}^{n} \sim x_{k}^{n}, 0 \leq k \leq n$.

It is clear that $X$ is a connected one-dimensional reducible analytic space. A holomorphic function $f$ on $X$ can be identified with a family $\left(f_{n}\right)_{n \geq 0}$ of holomorphic functions $f_{n}$ on $D_{n}, n \geq 0$, such that $f_{n}\left(x_{k}^{n}\right)=f_{0}\left(q_{k}^{n}\right), n>0$, $0 \leq k \leq n$.

Let $O_{n}$ be the image in $X$ of the origin $O$ of $D_{n}, n \geq 0$.

Theorem. (a) The Carathéodory pseudodistance $c_{X}$ is a distance on $X$, it defines the topology of $X$, and the space $X$ is $c_{X}$-complete.

(b) There exists $r>0$ such that the Caratheodory ball $B_{c_{X}}\left(O_{0}, r\right)$ is not relatively compact in $X$.

Observe that by the Hurwitz theorem we have the following:

Lemma 1. Let

$$
f_{n}(\lambda):=\prod_{k=0}^{n} \frac{\lambda-p_{k}^{n}}{1-\overline{p_{k}^{n}} \lambda}, \quad \lambda \in \Delta
$$

Suppose that $f_{n_{j}} \rightarrow f$ locally uniformly on $\Delta$. Then $|f(0)|=1 / e$ and $f$ is without zeros on $\Delta$.

The natural isomorphism $i_{n}: D_{n} \rightarrow \Delta$ induces a holomorphic mapping $p$ : $X \rightarrow D_{0}$.

Lemma 2. Let $K \subset \subset \Delta$, and let $N_{0}$ be an integer such that $p_{k}^{n} \notin K, n \geq N_{0}$, $0 \leq k \leq n$. Denote by $X_{N_{0}}$ the union of the images in $X$ of the $D_{n}, n \geq N_{0}$. Then there exists a constant $C>0$ such that, whenever $x, y \in X_{N_{0}}, x \neq y$, with $p(x)=p(y) \in K$, then $c_{X}(x, y)>C$.

Proof. Suppose that $c_{X}\left(x_{\nu}, y_{\nu}\right) \rightarrow 0$, where $x_{\nu}, y_{\nu} \in X, x_{\nu} \neq y_{\nu}$, and $p\left(x_{\nu}\right)=p\left(y_{\nu}\right) \in K$. Since bounded holomorphic functions on $X$ separate points of $X$, we can assume that $x_{\nu} \in D_{n(\nu)}$ with $n(\nu) \rightarrow \infty$.

Observe that $F_{n}$ with $F_{n}(x):=0, x \in D_{m}, m \neq n$, and $F_{n}(x):=f_{n}(x)$, $x \in D_{n}$, is holomorphic on $X$. Therefore, since $c_{X}\left(x_{\nu}, y_{\nu}\right) \rightarrow 0$, we get that $f_{n(\nu)}\left(x_{\nu}\right) \rightarrow 0$, which contradicts Lemma 1.

Now, we pass to the proof of the theorem.

Proof. Assertion (a) follows directly from Lemma 2. In order to prove (b), let $g: X \rightarrow \Delta$ be holomorphic with $g\left(O_{0}\right)=0$. Define on $\Delta$

$$
\varphi_{n}(\lambda):=\frac{1}{2}\left(g\left(\lambda_{n}\right)-g\left(\lambda_{0}\right)\right),
$$

where $\lambda_{k} \in X$ denotes the image in $X$ of $\lambda \in D_{k}, k \geq 0$.

It is clear that $\varphi_{n} \in \mathscr{O}(\Delta, \Delta)$ and that $\varphi_{n}\left(p_{k}^{n}\right)=0,0 \leq k \leq n$. Therefore, by the Schwarz Lemma, we obtain that

$$
\left|\varphi_{n}(0)\right| \leq\left(1-\frac{1}{1+n}\right)^{n+1},
$$


which gives $\left|g\left(O_{n}\right)\right| \leq 2\left(1-\frac{1}{1+n}\right)^{n+1}$. Hence $O_{n} \in B_{c_{X}}\left(O_{0}, r\right), r:=\omega\left(0, \frac{4}{5}\right)$, if $n \gg 1$.

Observe that $B_{c_{X}}\left(O_{0}, r\right)$ is disconnected and has relatively compact components. Therefore the closure of $B_{c_{X}}\left(O_{0}, s\right)$ is not equal to the closed Carathéodory ball $\left\{y \in X: c_{X}\left(O_{0}, y\right) \leq s\right\}$ for some $s$ (cf. [3]).

In order to conclude we recall that, so far, there is no example of an analytic space satisfying (a) of the theorem but which is not $H^{\infty}$-convex.

\section{REFERENCES}

1. M. Jarnicki and P. Pflug, Invariant pseudodistances-completeness and product property, Ann. Polon. Math. 55 (1991), 169-189.

2. M. Jarnicki, P. Pflug, and J.-P. Vigué, The Carathéodory distance does not define the topology-the case of domains, C. R. Acad. Sci. Paris Sér. I Math. 312 (1991), 77-79.

3. _ A remark on Carathéodory balls, Arch. Math. 58 (1992), 595-598.

4. S. Kobayashi, Intrinsic distances, measures and geometric function theory, Bull. Amer. Math. Soc. 82 (1976), 357-416.

5. M. A. Selby, On completeness with respect to the Carathéodory metric, Canad. Math. Bull 17 (1974), 261-263.

6. N. Sibony, Prolongement des fonctions holomorphes bornées et métrique de Carathéodory, Invent. Math. 29 (1975), 205-230.

7. J.-P. Vigué, The Carathéodory distance does not define the topology, Proc. Amer. Math. Soc. 91 (1984), 223-224.

Uniwersytet Jagielloński, Instytut Matematyki, Reymonta 4, 30-059 Kraków, Poland E-mail address: UMJARNIC@PLKRCY11.BITNET

Universität Osnabrück, Standort Vechta, Fachbereich Naturwissenschaften, Mathematik, Postfach 15 53, 2848 Vechta, Germany

E-mail address: PFLUGVEC@DOSUNI1.BITNET

Universite de Poitiers, Mathématiques, 40, Avenue du Recteur Pineau, 86022 Poitiers Cedex, France

E-mail address: VIGUE@FRUPTS51.BITNET 